\title{
Errors in calculated planetary phase functions and albedos due to neglecting polarization
}

\author{
D. M. Stam and J. W. Hovenier
}

\author{
Astronomical Institute "Anton Pannekoek", University of Amsterdam, Kruislaan 403, 1098 SJ Amsterdam, The Netherlands \\ e-mail: dstam@science.uva.nl
}

Received 27 June 2005 / Accepted 13 August 2005

\begin{abstract}
Accurate calculations of disk-integrated quantities such as planetary phase functions and albedos will be crucial for the analysis of direct observations of light that is reflected by extrasolar planets. We show that adopting a scalar representation of light and thus neglecting the polarized nature of light leads to significant, wavelength dependent, errors in calculated planetary phase functions and geometric albedos of homogeneous giant planets. The errors depend on the planetary model atmosphere. For planets with little to no aerosol/cloud particles in their atmosphere, these errors can reach more than $9 \%$. For cloud covered planets, the errors are generally smaller, but can still reach several percent. The errors in the planetary phase function and geometric albedo also depend on the atmospheric absorption optical thickness. Neglecting polarization thus influences the absolute and relative depth of absorption bands in albedos and phase functions, and thus indirectly e.g. a gaseous mixing ratio that is derived from the depth of a band. Specifically, we find that neglecting polarization when deriving the methane mixing ratio from numerically simulated reflection spectra of a giant planet can lead to values that are too large by several tens of percent. Neglecting polarization generally leads to (wavelength and absorption optical thickness dependent) errors smaller than $0.5 \%$ in calculated (monochromatic) planetary Bond albedos. The errors in phase functions and albedos due to neglecting only circular polarization appear to be smaller than $0.0005 \%$. When calculating phase functions and albedos of homogeneous planets, describing light by its intensity (or flux) and its state of linear polarization should thus suffice.
\end{abstract}

Key words. techniques: polarimetric - stars: planetary systems - polarization - radiative transfer

\section{Introduction}

Recently, the first direct observations of extrasolar planets have been reported (Chauvin et al. 2004, 2005; Charbonneau et al. 2005; Deming et al. 2005). After the flurry of indirect planet detections, in which careful observations of the motion of a star or its flux reveal the presence of a substellar companion, the extremely challenging direct observations, in which radiation from the planet itself is observed, are a crucial next step in the research of extrasolar planets. In the first place, direct observations can help establish the orbital inclination angle of the vast majority of extrasolar planet candidates that have so far only been detected through radial velocity measurements of their star. With this orbital inclination angle, the mass of a planetary candidate can be determined and with that its true nature (a planet or e.g. a brown dwarf). Second, direct observations enable the detection of planets that are beyond the reach of indirect detections methods, such as small, terrestrial planets. Third, while indirect observations are very useful to detect a planet, they do not provide much information about the physical characteristics of the planet, such as its atmospheric composition and structure, the presence of clouds, and the surface properties. To derive such physical characteristics, direct observations, i.e. observations of the thermal radiation the planet emits and/or the starlight the planet reflects are required.

From direct observations of planetary radiation, the physical characteristics of a planet can generally be derived by comparing the observations with results of numerical simulations for various planetary models. In this paper, we concentrate on numerical simulations of starlight that is reflected by a planet (from visible to near-infrared wavelengths) and discuss the errors in calculated wavelength dependent planetary phase functions, geometric albedos and Bond (or spherical) albedos that are due to describing light by a scalar instead of by a vector, thus neglecting the polarized nature of light.

People that perform radiative transfer calculations are generally aware of the fact that a full description of light comprises not only its intensity or flux, but also its state of polarization. A proper vector description of light is, however, usually applied only when the state of polarization itself is calculated; when intensities or fluxes are calculated, light is mostly approximated by a scalar. Reasons for using this approximation are probably that numerical radiative transfer algorithms that include polarization are much more complex than those using only scalars, and radiative transfer calculations take much more time to run when vectors are used instead of scalars. In addition, people usually do not realise that neglecting polarization leads to 


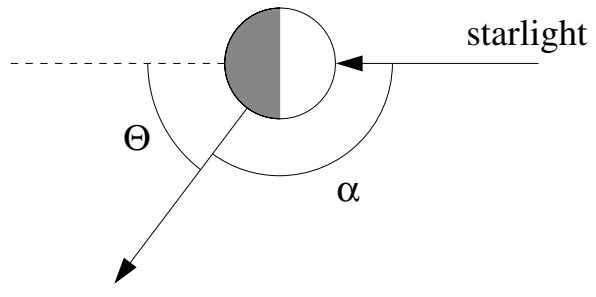

to observer

Fig. 1. Sketch of the geometry: $\Theta$ is the total scattering angle, and $\alpha$ is the planetary phase angle, i.e. the angle between the star and the observer as seen from the center of the planet.

errors in calculated fluxes, or they simply assume these errors are negligible. Using the results presented in this paper, people can either decide that they should take polarization into account (see e.g. Hovenier et al. 2004) or estimate the errors in their results.

Errors in the intensity and/or flux of light scattered within planetary atmospheres due to neglecting polarization have been presented before: Chandrasekhar (1950); van de Hulst (1980) and Mishchenko et al. (1994) presented intensity errors for model atmospheres containing only gaseous molecules, whereas Hansen (1971) and Lacis et al. (1998), and Sromovsky (2005b) discussed errors for, respectively, Earth-like and Neptune-like model atmospheres containing both gaseous molecules and aerosol/cloud particles. Most of the errors presented before pertain to light reflected by and/or light transmitted through plane-parallel model atmospheres and thus apply to observations of a planet's atmosphere with fairly high spatial resolution, aiming at e.g. remote-sensing of the Earth's atmosphere from a satellite at an altitude of about $400 \mathrm{~km}$, or Hubble Space Telescope (HST) observations of Neptune (Sromovsky 2005b).

In this paper, we are mostly interested in numerical simulations of direct observations of extrasolar planets. These planets will, for years to come, present themselves as a speck of light without any spatially resolved features. We therefore study the errors that result when starlight that is reflected by a locally plane-parallel planetary atmosphere is integrated over the illuminated and visible part of the planetary disk. We thus integrate the reflected light over local angles of incidence ranging from $0^{\circ}$ to $90^{\circ}$ and local angles of reflection ranging from $0^{\circ}$ to $90^{\circ}$ (when $90^{\circ} \leq \Theta \leq 180^{\circ}$ ) or from $90^{\circ}-\Theta$ to $90^{\circ}$ (when $\Theta<90^{\circ}$ ), with $\Theta$ the total scattering angle (see Fig. 1). This total scattering angle equals $180^{\circ}-\alpha$, with $\alpha$ the planetary phase angle, i.e. the angle between the star and the observer as viewed from the center of the planet.

We will present wavelength dependent planetary phase functions, geometric and Bond albedos and the errors in them that are due to neglecting polarization for total scattering angles $\Theta$ ranging from $0^{\circ}$ (all of the planet's nightside is in view) to $180^{\circ}$ (all of the planet's dayside is in view). Obviously, at which values of the total scattering angles an extrasolar planet in a given planetary system will in theory be observable depends on the inclination angle, $i$, of the planetary orbit, i.e. the angle between the normal on the orbit and the direction towards the observer. Namely, in case the planetary orbit is seen face-on $\left(i=0^{\circ}\right), \Theta$ is always equal to $90^{\circ}$, while in case the orbit is seen edge-on $\left(i=90^{\circ}\right), \Theta$ ranges from $0^{\circ}$ to $180^{\circ}$ along the orbit (see Stam et al. 2004). Note that, as seen from the Earth, the angular distance between an extrasolar planet and its star will always be extremely small. Hence, the chances of spatially separating an extrasolar planet from its star are thus largest for values of $\Theta$ (and $\alpha$ ) around $90^{\circ}$.

For our numerical simulations, we concentrate on Jupiterlike, gaseous model planets with and without cloud layers, since giant planets are the most obvious candidates for the first direct observations. The atmospheres of our extrasolar model planets contain methane, just like the giant planets in our own solar system. Methane leaves distinct absorption bands throughout the visible to near-infrared wavelength range (see e.g. the observations of the solar system giant planets by Karkoschka 1994). Using numerical simulations, we will discuss the effects of neglecting polarization on the shape (width and depth) of the methane absorption bands. Such effects are to be expected because the errors due to neglecting polarization generally depend on the single scattering albedo of the atmospheric constituents, as also shown by Mishchenko et al. (1994) and Sromovsky (2005b). Obviously, accurate calculations of the shape of gaseous absorption bands, such as those of methane, are in particular important when trying to derive the mixing ratio and/or vertical distribution of the absorbing gas from observations, and for deriving e.g. cloud top altitudes.

Although we aim our numerical simulations of reflected fluxes and the errors due to neglecting polarization at direct observations of extrasolar planets, our results are also useful for disk-integrated observations of planets within our own solar system. In particular, our numerical simulations regarding geometric albedos are suitable for Earth-based observations (either from the ground or from an Earth-centered orbit) of the solar system's giant planets, like the simulations presented by Sromovsky (2005b), because such observations can only be performed when $\Theta$ is close to $180^{\circ}$ (or, $\alpha$ close to $0^{\circ}$ ).

In Sect. 2, we describe the treatment of light as a vector instead of as a scalar as well as our radiative transfer algorithm. In Sect. 3, we present the errors in planetary phase functions and albedos that are due to neglecting polarization. For a planet with a clear atmosphere, i.e. an atmosphere without aerosol and/or cloud particles, we study the dependence of the errors on the atmospheric molecular scattering optical thickness. For both clear and cloudy Jupiter-like model atmospheres, we study the dependence of the errors on the wavelength, in particular the influence of neglecting polarization on the shape of methane absorption bands. In Sect. 4, we summarize and discuss our results.

\section{Describing and calculating reflected starlight}

\subsection{Stokes vectors}

The flux and state of polarization of light can fully be described by a Stokes (column) vector (see Hovenier et al. 2004)

$\pi \boldsymbol{F}=\pi[F, Q, U, V]$,

which we call the flux vector. Here, Stokes parameter $F$ is the total flux divided by $\pi, Q$ and $U$ describe the linearly polarized 
flux, and $V$ the circularly polarized flux. Fluxes and Stokes parameters are expressed in $\mathrm{W} \mathrm{m}^{-2} \mathrm{~Hz}^{-1}$. Stokes parameters $Q$ and $U$ are defined with respect to a reference plane, for which we chose the so-called planetary scattering plane, i.e. the plane through the centers of the star and the planet, that also contains the observer.

The flux vector of starlight that has been reflected by a spherical, horizontally homogeneous planet with radius $r$, that is observed at distance $d$ (with $d \gg r$ ) is given by (see Stam et al. 2004)

$\pi \boldsymbol{F}(\Theta)=\frac{r^{2}}{d^{2}} \frac{1}{4} \mathbf{S}(\Theta) \pi \boldsymbol{F}_{0}$.

Here, $\Theta$ is the total scattering angle $\left(0^{\circ} \leq \Theta \leq 180^{\circ}\right.$ ) (see Fig. 1). $\mathbf{S}$ is the planetary scattering matrix, which describes the light that has been scattered within the planetary atmosphere and that has been reflected towards the observer (see Stam et al., in preparation), and $\pi \boldsymbol{F}_{0}$ is the flux vector describing the starlight that is incident on the planet, with $\pi F_{0}$ the stellar flux (in $\mathrm{W} \mathrm{m}^{-2} \mathrm{~Hz}^{-1}$ ) measured perpendicular to the incoming direction. The total scattering angle $\Theta$ equals $180^{\circ}-\alpha$, with $\alpha$ the planetary phase angle. Unless otherwise stated, we will use $\Theta$ instead of $\alpha$ in this article.

\subsection{The planetary phase function}

Using the planetary scattering plane as the reference plane, and assuming the planet is mirror-symmetric with respect to this reference plane, matrix $\mathbf{S}$ can be described by (see Stam et al., in preparation)

$\mathbf{S}(\Theta)=\left[\begin{array}{cccc}a_{1}(\Theta) & b_{1}(\Theta) & 0 & 0 \\ b_{1}(\Theta) & a_{2}(\Theta) & 0 & 0 \\ 0 & 0 & a_{3}(\Theta) & b_{2}(\Theta) \\ 0 & 0 & -b_{2}(\Theta) & a_{4}(\Theta)\end{array}\right]$.

Element $a_{1}$ of this matrix is generally referred to as the planetary phase function.

The non-zero elements of $\mathbf{S}$ clearly show that in case the incoming stellar light $\pi \boldsymbol{F}_{0}$ were polarized in the sense that $Q_{0} \neq 0$ (with respect to the planetary scattering plane), the flux $\pi F$ of the reflected starlight would be proportional to $a_{1} F_{0}+b_{1} Q_{0}$. An accurate calculation of $\pi F$ would thus require a vector instead of a scalar description of the starlight (note that $b_{1}$ can be several tens of percents of $a_{1}$ (see e.g. Stam et al. 2004)).

In reality, light of a solar-type star can be assumed to be unpolarized when integrated over the stellar disk (Kemp et al. 1987). Flux vector $\pi \boldsymbol{F}_{0}$ is thus given by the column vector $\pi F_{0}[1,0,0,0]$, and in case one wants to calculate the reflected flux $\pi F$, one only needs element $a_{1}$ of $\mathbf{S}$. It may thus seem that the scalar description of light works just fine. However, the calculation of $a_{1}$ itself still requires the vector description of light. Single scattering processes within a planetary atmosphere that contains homogeneous, spherical symmetric particles, or irregularly shaped, randomly oriented particles are namely described by scattering matrices that are similar to the planetary scattering matrix in Eq. (3) (see e.g. Hovenier et al. 2004). Unpolarized sunlight that is incident on the planetary atmosphere will thus generally become polarized when it is scattered by a gaseous molecule or aerosol/cloud particle in the planetary atmosphere. For each subsequent scattering event, the scattered intensity will thus depend on the state of polarization of the incoming light. Because the planetary phase function $a_{1}$ comprises not only the singly scattered light that is reflected by the planet, but also the multiple scattered light, a vector description of light should thus be used in its calculation, even if in the end one is only interested in calculating the reflected flux.

\subsection{The Bond albedo and the geometric albedo}

In principle, polarization should not only be included in the calculation of the planetary phase function $a_{1}$, but also in derivative quantities, such as the planet's Bond albedo and its geometric albedo.

A planet's (monochromatic) Bond albedo, $A_{\mathrm{B}}$, is defined as the fraction of incident stellar flux that is reflected by the planet for incident unpolarized light with wavelength $\lambda$, and is also referred to as the (monochromatic) spherical albedo. The Bond albedo is important for e.g. determining a planet's energy balance, since it indicates how much energy the planet absorbs. For the latter application, the Bond albedo generally pertains to spectral regions, e.g. visible wavelengths, rather than to the single wavelength we use here.

The Bond albedo equals the average of $a_{1}$ over all directions, thus

$A_{\mathrm{B}}=\frac{1}{4 \pi} \int_{4 \pi} a_{1}(\Theta) \mathrm{d} \omega$

where $\mathrm{d} \omega$ is an element of solid angle. Note that in case there is no absorption in a planetary atmosphere and no absorbing surface below the atmosphere, neglecting polarization in the computation of $a_{1}$ should not lead to an error in a planet's Bond albedo, because in that case all the light that is incident on the planet will be reflected back to space anyhow, and the Bond albedo should equal 1.0 with or without taking polarization into account.

For observers, a planet's geometric albedo $A_{\mathrm{G}}$ is more relevant than $A_{\mathrm{B}}$, because $A_{\mathrm{G}}$ indicates a planet's brightness when it is in opposition or in superior conjunction. We have

$A_{\mathrm{G}}=\frac{1}{4} a_{1}\left(180^{\circ}\right)$.

Obviously, the errors in $A_{\mathrm{G}}$ due to neglecting polarization are directly related to the errors in the planetary phase function $a_{1}$ at $\Theta=180^{\circ}$.

\subsection{Radiative transfer calculations}

The modeling errors in the phase function and the Bond albedo of a planet with a given model atmosphere that are due to neglecting polarization will depend on the contribution of multiply scattered light (second and higher orders) to the reflected starlight, and on the vertical distribution of the atmospheric particles and their single scattering properties. We describe a planetary atmosphere as a locally plane-parallel stack of horizontally homogeneous layers that may be different from each other, bounded below by a black surface (i.e. light that escapes 
the atmosphere at the bottom is absorbed). Each layer contains gaseous molecules, and, optionally, aerosol/cloud particles.

Light scattering by molecules is described by anisotropic Rayleigh scattering (see Hansen \& Travis 1974; Stam et al. 2002). For the depolarization factor that is included in the single scattering matrix describing anisotropic Rayleigh scattering, we choose the value for $\mathrm{H}_{2}$, the main constituent of atmospheres of giant, Jupiter-like planets, i.e. 0.02. The value of the depolarization factor will influence the errors that are due to neglecting polarization. For plane-parallel, purely molecular model atmospheres, this influence has been investigated extensively by Mishchenko et al. (1994). In Sect. 4, we will discuss the effects of the depolarization factor on our results. Given their size distribution and refractive index, the optical properties of aerosol/cloud particles are calculated using Mie-theory (van de Hulst 1957; de Rooij \& van der Stap 1984). We thus assume these particles to be homogeneous and spherical.

For the radiative transfer calculations, we have to describe for each atmospheric layer: its optical thickness, the single scattering albedo and the scattering matrix of the mixture of molecules and aerosol/cloud particles. We assume all scattering processes to be elastic, and thus ignore the wavelength redistribution that is due to an inelastic scattering process like Raman scattering (see e.g. Stam et al. 2002; Sromovsky 2005a).

Given a planetary model atmosphere as described above, the planetary phase function $a_{1}$ for $\Theta$ ranging from $0^{\circ}$ to $180^{\circ}$ (see Eq. (3)) and the planetary Bond albedo are calculated using a combination of 1) an adding-doubling radiative transfer algorithm to calculate the locally reflected starlight (de Haan et al. 1987); and 2) an algorithm to integrate the locally reflected starlight over the illuminated part of the planetary disk (Stam et al. 2005, in preparation).

Both the adding-doubling algorithm and the diskintegration algorithm use Gaussian integration procedures, the accuracy of which depends on the number of Gaussian abscissae that is used. The number of Gaussian abscissae that is required to reach a given accuracy depends on the structure and composition of the model atmosphere, as well as on the total scattering angle $\Theta$ (generally, the larger the scattering particles and the smaller $\Theta$, the more abscissae are required, see Stam et al. 2005, in preparation). For the calculations presented in this paper, we made sure to choose the number of abscissae large enough so that the numerical errors in the calculated fluxes were much smaller than the errors due to neglecting polarization at all values of $\Theta$. It appeared that for the most complex model atmosphere used in this paper, namely that with a cloud layer (see Sect. 3.2.2), the 64 Gaussian abscissae that we employed were more than sufficient across the whole phase angle range.

The modeling errors due to neglecting polarization are obtained by comparing planetary phase functions, geometric albedos and Bond albedos calculated using $1 \times 1$ scattering matrices (the scalar case, thus ignoring polarization), with results calculated using $3 \times 3$ matrices (taking linear polarization into account, but neglecting circular polarization), and $4 \times 4$ matrices (the full description of polarized light). For model atmospheres in which only (isotropic) Rayleigh scattering takes place, there is no difference between fluxes calculated with $3 \times 3$

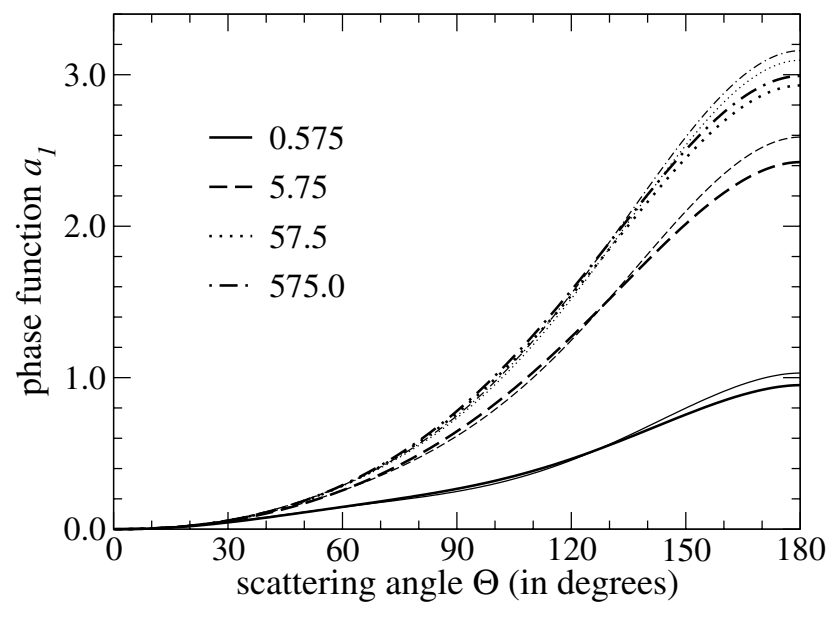

Fig. 2. The phase functions of a planet with a non-absorbing molecular model atmosphere of (scattering) optical thickness 0.575 (solid lines), 5.75 (dashed lines), 57.5 (dotted lines), and 575.0 (dot-dashed lines) calculated neglecting polarization, thus using $1 \times 1$ matrices (thick lines), and taking polarization into account, thus using $3 \times 3$ matrices (thin lines).

and $4 \times 4$ matrices because of the shape of the Rayleigh scattering matrix (see Hansen \& Travis 1974; Stam et al. 2002). For model atmospheres that contain aerosol and/or cloud particles, neglecting circular polarization (thus using $3 \times 3$ matrices) will generally lead to errors, albeit very small ones as we will show in Sect. 3.2.2.

In this paper, absolute errors due to neglecting polarization will be calculated as $\left(X_{3 \times 3}-X_{1 \times 1}\right)$ or $\left(X_{4 \times 4}-X_{1 \times 1}\right)$, with $X$ the planetary phase function, the geometric albedo or the Bond albedo at a given value of $\Theta$ and/or $\lambda$. The subscripts $1 \times 1,3 \times 3$, or $4 \times 4$, indicate the size of the matrices that are used in the radiative transfer calculations. Relative errors due to neglecting linear and/or circular polarization are calculated as the absolute errors divided by $X_{1 \times 1}$. Negative error values thus indicate that neglecting polarization results in a planetary phase function or albedo that is too large, whereas positive error values indicate that neglecting polarization results in a phase function or albedo that is too small.

\section{The modeling errors}

\subsection{Dependence on the atmospheric optical thickness}

To study how the errors that are due to neglecting polarization depend on the atmospheric optical thickness, $b$, we use a model atmosphere that consists of a single layer containing only nonabsorbing gaseous molecules. The single scattering albedo in the atmospheric layer is thus 1.0. The (scattering) optical thickness of this atmosphere is varied between $10^{-2}$ and $10^{3}$.

Figure 2 shows the phase function $a_{1}$ (see Eq. (3)) of our model planet for four values of the atmospheric optical thickness: $0.575,5.75$ (which is representative for the upper part of a Jupiter-like atmosphere at $\lambda=0.55 \mu \mathrm{m}$ ), 57.5, and 575.0. For each atmospheric optical thickness $b$, we have plotted two lines: one for calculations in which polarization is neglected, and the other for calculations in which the polarization is fully 

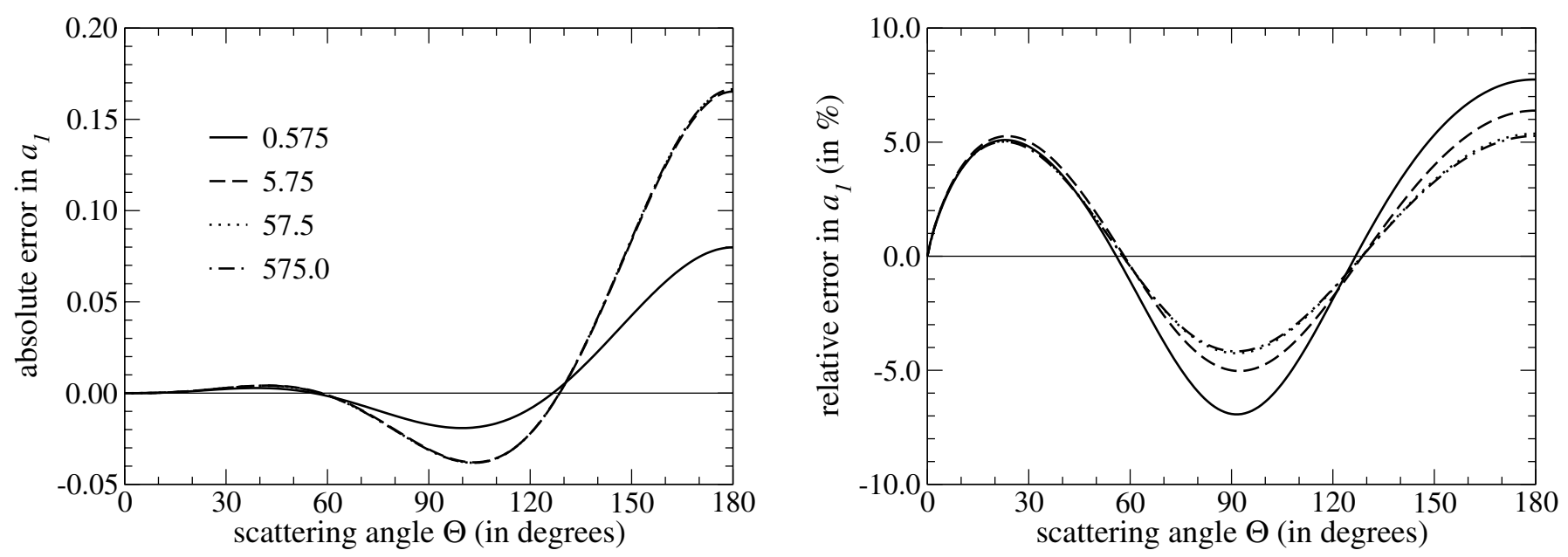

Fig. 3. The errors in the phase functions of Fig. 2 when polarization is neglected, thus when $1 \times 1$ matrices are used instead of $3 \times 3$ matrices: a) the absolute errors, and $\mathbf{b}$ ) the relative errors. The errors are shown for atmospheric (scattering) optical thicknesses $b$ of 0.575 (solid line), 5.75 (dashed line), 57.5 (dotted line), and 575.0 (dot-dashed line). There is no absorption in the atmosphere. Note that the absolute errors for $b \geq 5.75$ and the relative errors for $b \geq 57.5$ are virtually indistinguishable.

accounted for. From Fig. 2 it is clear that the phase functions that are calculated taking polarization into account, differ significantly from those calculated without polarization. Thus, according to Eq. (2), reflected fluxes calculated with polarization will differ significantly from those calculated without polarization. Because the planetary geometric albedo $A_{\mathrm{G}}$ is proportional to $a_{1}\left(180^{\circ}\right)$, Fig. 2 also shows that ignoring polarization leads to much lower values of $A_{\mathrm{G}}$ than when polarization is properly taken into account.

Figure 3 gives a more quantitative view of the errors that occur in the phase functions of Fig. 2 in case polarization is neglected. Figure $3 \mathrm{a}$ shows the absolute errors and Fig. $3 \mathrm{~b}$ the relative errors for the four atmospheric optical thicknesses used in Fig. 2. Clearly, both the absolute and the relative errors depend strongly on the total scattering angle $\Theta$ : the errors are generally largest (in absolute value) at the largest values of $\Theta$ and smallest (in absolute value) at the smallest $\Theta$ 's. Because of the total scattering angle dependence of the errors, neglecting the polarization in the calculation of the planet's phase function influences the distribution of the reflected flux across the illuminated half of the planet. For example, when polarization is accounted for, the planet is brighter at $\Theta=180^{\circ}$ and at $\Theta \approx 20^{\circ}$ (see Fig. 3b), and darker at $\Theta \approx 90^{\circ}$, than when polarization is neglected. A similar strong total scattering angle dependence was found by Mishchenko et al. (1994) for purely molecular, plane-parallel atmospheres, thus without integrating the reflected light over the illuminated and visible part of a planetary disk. In case of a plane-parallel atmosphere, the total scattering angle thus simply refers to the angle between the direction of propagation of the incident light and that of the reflected light.

The errors plotted in Fig. 3 tend to zero as $\Theta$ approaches zero, because at small total scattering angles most of the light that is reflected by the planet has been scattered only once, as shown by Hovenier \& Stam (2005). Because the incident light is unpolarized, this singly scattered light leaves no error due to neglecting polarization. The large errors at total scattering angles near $100^{\circ}$ (Fig. 3a) and $90^{\circ}$ (Fig. 3b) and $180^{\circ}$ can be attributed to the high degree of polarization of the first order scattered light that is scattered over a single scattering angle of $90^{\circ}$ and that is the source for a large fraction of the second order scattered light at these total scattering angles (Mishchenko et al. 1994).

Besides on the total scattering angle $\Theta$, the absolute and relative errors in our planetary phase functions depend also on the atmospheric (scattering) optical thickness $b$ : across most of the total scattering angle interval in Fig. 3, the absolute errors increase (in absolute value) with increasing $b$, while the relative errors decrease (in absolute value) with increasing $b$. Interestingly, for each total scattering angle, the errors can be seen to converge with increasing $b$ in both Figs. $3 a$ and $3 b$. In particular, for the optical thicknesses used in Fig. 3, the absolute errors appear to reach convergence for $b \geq 5.75$, and the relative errors for $b \geq 57.5$.

The absolute errors in the phase functions converge with increasing $b$ mainly because deep layers in the atmosphere receive little light from the upper atmospheric layers, and their contribution to the light that emerges from the top of the atmosphere (i.e. the light that is reflected back to space) is thus small. Hence, increasing the (scattering) optical thickness $b$ of an already thick atmosphere leads neither to significant increases of the total flux of reflected light (which is also apparent from the convergence of the reflected fluxes in Fig. 2) nor to a significant increase of the error in this flux due to neglecting polarization. The convergence of the relative errors in the planetary phase functions with increasing $b$ comes forth simply from the convergence of the absolute errors and the convergence of the total reflected flux.

The errors plotted in Fig. 3 are generally largest (in absolute value) at the largest total scattering angle, i.e. at $\Theta=180^{\circ}$. Since $\frac{1}{4} a_{1}\left(180^{\circ}\right)=A_{\mathrm{G}}$, the planetary geometric albedo $A_{\mathrm{G}}$ is apparently very sensitive to neglecting polarization. It is important to note that at $\Theta=180^{\circ}$, the degree of polarization of the reflected light equals zero (see e.g. Stam et al. 2004) which 


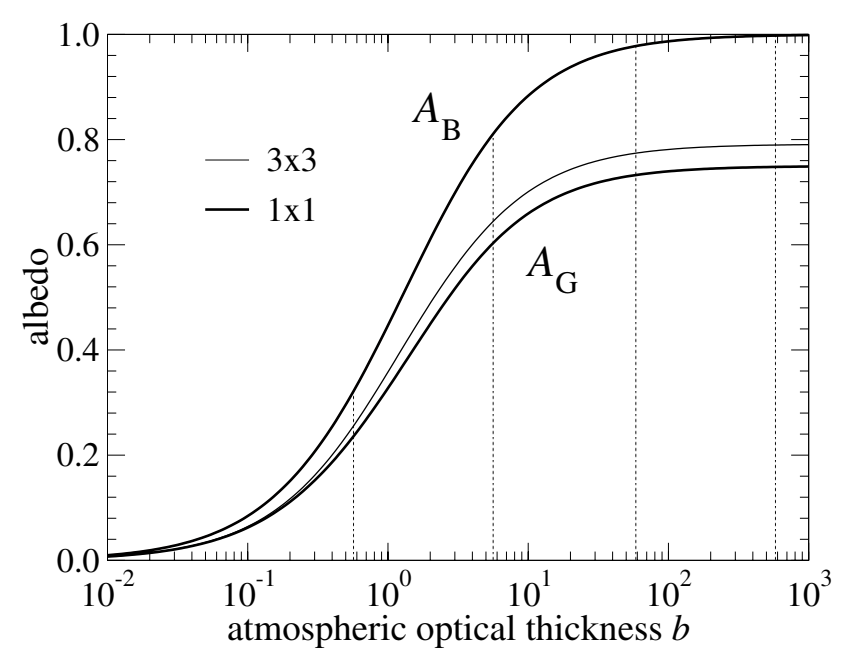

Fig. 4. The geometric albedos, $A_{\mathrm{G}}$, and the Bond albedos, $A_{\mathrm{B}}$, of a planet with non-absorbing molecular model atmosphere as functions of the atmospheric (scattering) optical thickness $b$, calculated neglecting polarization (thick lines), and taking polarization into account (thin lines). The thick and thin lines pertaining to the Bond albedo are indistinguishable. The vertical dotted lines indicate the values of $b$ that were used in Figs. 2 and 3.

might lead people to wrongly assume that at and near this total scattering angle, polarization can safely be neglected. From Fig. 3, it can be derived that the absolute error in the geometric albedo $A_{\mathrm{G}}$ equals 0.02 for $b=0.575$ and about 0.04 for $b \geq 5.75$. The relative error in $A_{\mathrm{G}}$ decreases from about $8 \%$ for $b=0.575$ to slightly more than $5 \%$ for $b \geq 57.5$. A more complete coverage of the atmospheric optical thickness dependence of $A_{\mathrm{G}}$ and that of the absolute and relative errors in $A_{\mathrm{G}}$, is provided in Figs. 4 and 5, in which $b$ runs from $10^{-2}$ to $10^{3}$ (the single scattering albedo in the atmospheric layer is still 1.0). Figures 4 and 5 also contain results regarding the planetary Bond albedo, $A_{\mathrm{B}}$, which will be discussed later in this section.

The convergence of $A_{\mathrm{G}}$ with increasing $b$, both with and without taking polarization into account, can clearly be seen in Fig. 4. With increasing $b, A_{\mathrm{G}}$ tends to 0.7914 when polarization is taken into account, and to 0.7498 when polarization is neglected. These two values differ slightly from those calculated for a conservative scattering, semi-infinite atmosphere by Sromovsky (2005b), who found geometric albedos equal to, respectively, 0.7908 and 0.7496 . This difference is entirely due to the slightly different depolarization factor adopted by Sromovsky (2005b) (he uses 0.022, while we use 0.02).

The absolute errors in $A_{\mathrm{G}}$ due to neglecting polarization (Fig. 5a) are virtually zero when $b \approx 10^{-2}$, they increase smoothly with increasing $b$, and reach a maximum value of almost 0.042 for $b \gtrsim 3$. This value corresponds with that found by Sromovsky (2005b). The errors are small at the smallest values of $b$ because the amount of multiple scattering is negligible in such thin atmospheres (recall that polarization influences the fluxes only for second and higher orders of scattering, since we assume unpolarized incomnig radiation). The explanation for the increase and subsequent convergence of the absolute errors in $A_{\mathrm{G}}$ with increasing $b$ is similar to that for the absolute errors in $a_{1}$, which has been discussed above.

The relative errors in $A_{\mathrm{G}}$ (Fig. 5b) increase with increasing $b$ as long as $b \lesssim 1$. The errors reach a maximum value of almost $9 \%$ at $b=1.12$, then decrease again, to converge to a value of about $5.2 \%$ at large values of $b$. The decrease and subsequent convergence of the relative errors with increasing $b$ for $b \gtrsim 1$ reflects the slow convergence of $A_{\mathrm{G}}$ (Fig. 4) as compared to the convergence of the absolute error in $A_{\mathrm{G}}$ (Fig. 5a). The curve of $A_{\mathrm{G}}$ in Fig. $5 \mathrm{~b}$, has a similar shape as the curves pertaining to the geometric albedos of plane-parallel atmospheres with large values of the single scattering albedo and small values of the surface albedo shown by Mishchenko et al. (1994). According to Mishchenko et al. (1994), for a plane-parallel, purely molecular model atmosphere, the relative error is maximum for $b \lesssim 1$, which is close to but somewhat smaller than the value we find for our locally plane-parallel, but spherical planet.

Apart from the geometric albedo $A_{\mathrm{G}}$, Fig. 4 also shows the planetary Bond albedo $A_{\mathrm{B}}$ as a function of the atmospheric optical thickness $b$. Clearly, at small optical thicknesses, the Bond albedo is small because a significant fraction of the light that is incident on the planet reaches the surface, where it is absorbed. With increasing $b$, the amount of light that reaches the surface decreases. The fraction of light that the planet reflects back to space in all directions, i.e. the Bond albedo (see Eq. (4)), thus increases with increasing $b$, and converges to 1.0 for large values of $b$, where virtually no light reaches the surface.

In Fig. 4, the line that pertains to the calculation of the Bond albedo $A_{\mathrm{B}}$ with polarization is indistinguishable from the line that is calculated neglecting polarization. The absolute and relative errors in $A_{\mathrm{B}}$ due to neglecting polarization can be seen in Fig. 5. In order to make the errors in $A_{\mathrm{B}}$ visible on the same scale as the errors in the geometric albedo $A_{\mathrm{G}}$, we have multiplied them with 100 .

Figures 4 and 5 show that neglecting polarization when calculating $A_{\mathrm{B}}$ leads only to very small errors. The explanation for the negligible size of the errors is that when calculating the Bond albedo, the reflected flux is integrated over all outgoing directions, and whether or not polarization is taken into account, conservation of energy requires that the total (i.e. integrated over all directions) reflected flux equals the flux that is incident on the planet minus the energy that is absorbed. Thus, without any absorption, the errors in the Bond albedo due to neglecting polarization would vanish completely. With absorption (in our case by the black surface beneath the model atmosphere), the errors do not vanish, because neglecting polarization generally changes the angular distribution of the scattered light within the planetary atmosphere, as illustrated by the significant errors in the planetary phase function. As a result, when absorption is present, neglecting polarization will slightly change the total amount of energy that is absorbed by the planet and thus the planetary Bond albedo.

As can be seen in Fig. 5a, the absolute errors in $A_{\mathrm{B}}$ are close to zero for small values of $b$ as those in $A_{\mathrm{G}}$ because here, most of the reflected light is singly scattered light. With increasing $b$, the absolute error in $A_{\mathrm{B}}$ increases, like that in $A_{\mathrm{G}}$. However, instead of converging towards this maximum value, like the 

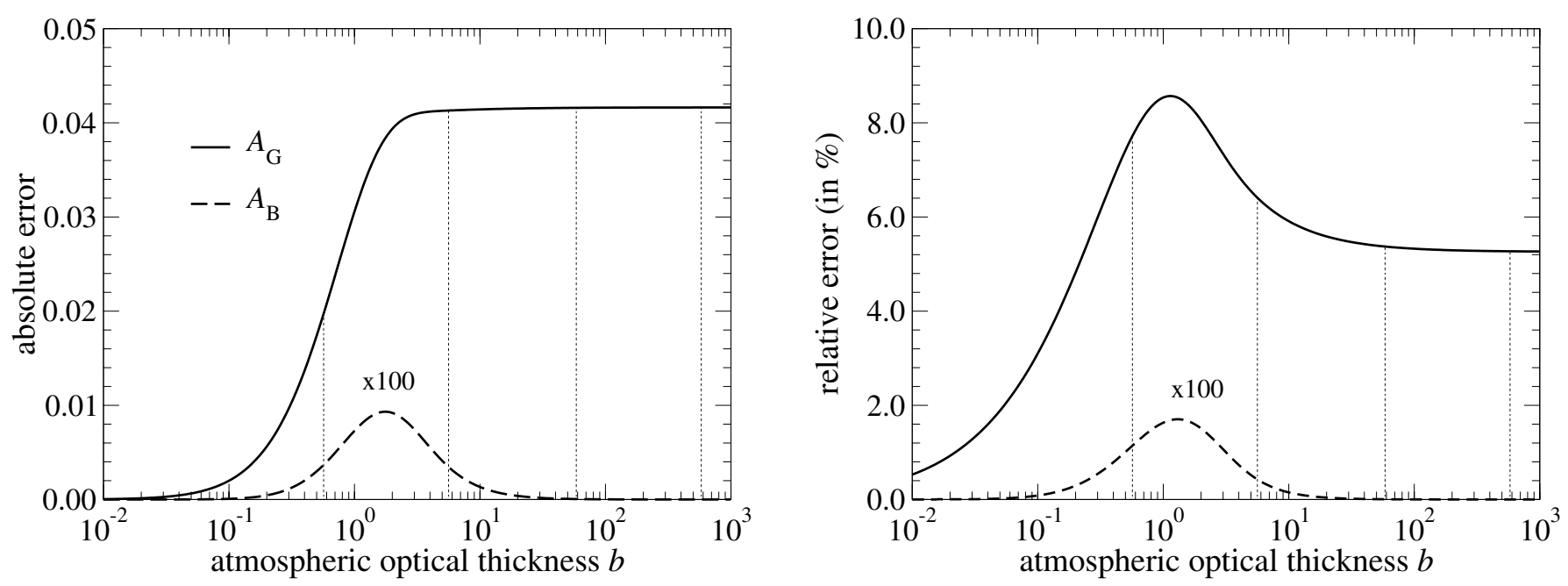

Fig. 5. The absolute a) and relative b) errors in $A_{\mathrm{G}}$ and $A_{\mathrm{B}}$ of Fig. 4 that are due to neglecting polarization. Both the absolute and the relative error in the Bond albedo $A_{\mathrm{B}}$ have been multiplied with 100 . The maximum absolute error in $A_{\mathrm{B}}$ is thus about 0.0001 , and the maximum relative error is about $0.017 \%$.

error in $A_{\mathrm{G}}$, the absolute error in $A_{\mathrm{B}}$ reaches a maximum value (i.e. 0.0001 at $b=1.74$ ) and then decreases again to zero for $b \gtrsim 30$. The explanation for this decrease is that for large values of $b$, the amount of light that emerges from the bottom of the atmosphere to be absorbed by the surface is very small. At these large values of $b$, virtually all the light that is incident on the planet is thus reflected back to space. The Bond albedo thus converges to 1.0 at large values of $b$, whether or not polarization is taken into account, and, consequently, the absolute error approaches zero.

The relative error in the Bond albedo (Fig. 5b) shows a similar optical thickness dependence as the absolute error, except that the relative error reaches its maximum value (i.e. $0.017 \%$ ) at a somewhat smaller value of $b$, namely 1.3 , due to the increase of $A_{\mathrm{B}}$ with increasing optical thickness (see Fig. 4).

\subsection{Dependence on the wavelength}

\subsubsection{A clear model atmosphere}

The albedo of single scattering and the optical thickness of a planetary atmosphere generally vary with wavelength. As a result, the errors due to neglecting polarization will vary with wavelength. In Fig. 6, we have plotted the wavelength dependent geometric albedo $A_{\mathrm{G}}$ and the Bond albedo $A_{\mathrm{B}}$ of a Jupiterlike planet as calculated with $(3 \times 3)$ and without $(1 \times 1)$ polarization. Like in Fig. 4, the line that pertains to the calculation of $A_{\mathrm{B}}$ taking polarization into account is indistinguishable from the line that is calculated neglecting polarization.

The Jupiter-like model planet has an atmosphere consisting of 38 plane-parallel, homogeneous layers, bounded below by a black surface. The ambient pressure ranges from $1.0 \times$ $10^{-4}$ bars at the top to 5.623 bars at the bottom of our model atmosphere ((Lindal 1992), supplemented from 1.0 to 5.623 bars by data from West et al. 1986). Across the wavelength region of our interest (from 0.4 to $1.0 \mu \mathrm{m}$ ), methane $\left(\mathrm{CH}_{4}\right)$ is the main absorbing gas in a Jupiter-like atmosphere. We assume an atmospheric mixing ratio of $\mathrm{CH}_{4}$ of $0.18 \%$, like on Jupiter,

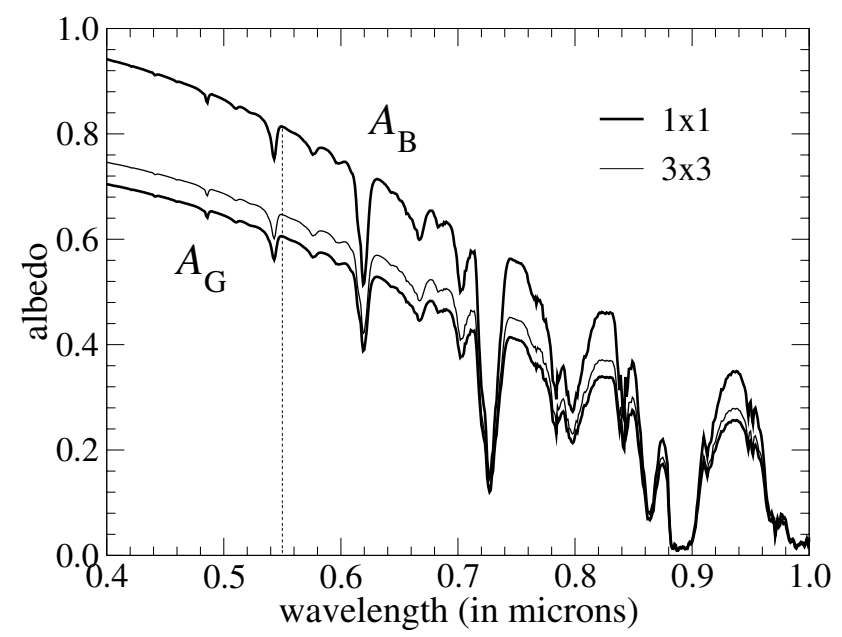

Fig. 6. The geometric albedo, $A_{\mathrm{G}}$, and the Bond albedo, $A_{\mathrm{B}}$, of a clear (i.e. without aerosol/cloud layers), Jupiter-like planet as functions of the wavelength, for $1 \times 1$ matrices (thick line) and $3 \times 3$ matrices (thin line). The thick and thin lines pertaining to the Bond albedo are indistinguishable. The vertical dotted line indicates the wavelength at which the atmospheric scattering optical thickness equals 5.75, the value used in Figs. 2 and 3.

and adopt the absorption cross-sections of Karkoschka (1994). Both the atmospheric scattering optical thickness and the atmospheric absorption optical thickness thus vary with wavelength. We used a depolarization factor of 0.02 , like before, and assumed this value to be wavelength independent across the wavelength range of our interest.

The model atmosphere used in the calculations for Fig. 6 contains no aerosol/cloud particles (using $3 \times 3$ matrices thus suffices for investigating the influence of neglecting polarization on reflected fluxes). The molecular scattering optical thickness of the model atmosphere decreases from 21.47 at $0.4 \mu \mathrm{m}$ to 0.51 at $1.0 \mu \mathrm{m}$. The vertical dashed line indicates the wavelength (i.e. $0.55 \mu \mathrm{m}$ ) at which the atmospheric molecular 


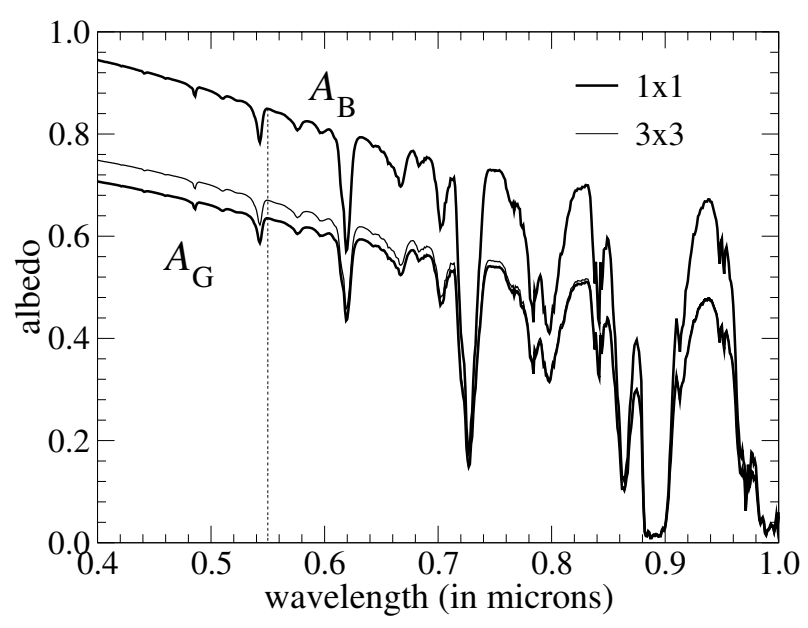

Fig. 7. Similar to Fig. 6, except with a cloud layer of scattering optical thickness 6.0 at $\lambda=0.55 \mu \mathrm{m}$ added to the deepest layers of the planetary model atmosphere.

scattering optical thickness equals 5.75, one of the values used in Figs. 2 and 3.

Not surprisingly in Fig. 6, in the continuum, i.e. outside gaseous absorption bands, both the geometric albedo $A_{\mathrm{G}}$ and the Bond albedo $A_{\mathrm{B}}$ of the model planet decrease with increasing wavelength because of the decreasing atmospheric scattering optical thickness in combination with the black surface below the atmosphere. In Fig. 6, one can see that in the continuum of $A_{\mathrm{G}}$, the absolute error due to neglecting polarization decreases slightly with increasing wavelength. This decrease of the error comes forth from the decrease of the scattering optical thickness of the atmosphere, and hence from the decrease of the amount of multiple scattering with increasing wavelength, in accordance with previous results (see Fig. 5a). More details on the wavelength dependence of the errors, in particular across the gaseous absorption bands, can be found in Fig. 8, where we have plotted the absolute and relative errors in $A_{\mathrm{G}}$ as a function of wavelength, both for the clear atmosphere and for a cloudy atmosphere (the latter will be discussed in Sect. 3.2.2).

Figure $8 \mathrm{a}$ indeed shows that for a clear model atmosphere, the absolute errors in the continuum decrease with increasing wavelength, although only slightly so at wavelengths smaller than $0.6 \mu \mathrm{m}$. For the clear model atmosphere, the relative errors in the continuum (Fig. 8b) increase with increasing wavelength up to $\lambda \approx 0.82 \mu \mathrm{m}$, where they start decreasing with increasing wavelength. The increase and the subsequent decrease of the relative errors in $A_{\mathrm{G}}$ is as expected from Fig. $4 \mathrm{~b}$, where the maximum error was found at an atmospheric scattering optical thickness of 1.12 (for the Jupiter-like model atmosphere used to derive the curves in Fig. 8b, the atmospheric scattering optical thickness is 1.12 at $\lambda=0.82 \mu \mathrm{m})$.

In the absorption bands of methane, the absolute error is smaller than in the adjacent continuum (see Fig. 8a). A straightforward explanation of this behaviour is the following: with increasing absorption, the amount of multiple scattering in the atmosphere decreases, and hence the error due to neglecting polarization decreases, since this error is only introduced when light is scattered more than once (assuming unpolarized incoming light). This explanation is at least valid for the absorption bands at the longest wavelengths, where the molecular scattering optical thickness of the atmosphere is small (recall that at $\lambda=0.82 \mu \mathrm{m}$, this scattering optical thickness equals 1.12). A similar explanation for decreasing errors with decreasing single scattering albedo is given by Mishchenko et al. (1994) for a thin, plane-parallel, model atmosphere.

For the absorption bands at the shortest wavelengths, where the atmospheric scattering optical thickness is large, a more subtle explanation for the error due to neglecting polarization is required. This can be inferred from the behaviour of the relative error across the absorption bands for the clear model atmosphere in Fig. 8b. Namely, while at wavelengths longwards of about $0.7 \mu \mathrm{m}$, the relative error in the absorption bands is smaller than in the adjacent continuum (which is perfectly in line with the above mentioned explanation of decreased multiple scattering), at shorter wavelengths, the relative error in the absorption bands is larger than in the adjacent continuum (note that when the atmospheric scattering optical thickness is large, a slight decrease of the multiple scattering due to absorption will have no significant effect). Thus, at the shorter wavelengths, neglecting polarization leads to bands that are slightly deeper than would be expected were the relative error constant across the band (note that in case the relative error were constant across an absorption band, the absolute error would still exhibit a wavelength dependence similar to that shown in Fig. 8a). The explanation for the increased depth of the absorption bands at the shortest wavelengths is the following: the dependence of the errors in the planetary phase function on $\Theta$ (Fig. 3) already showed that neglecting polarization leads to a different angular distribution of the light than when polarization is properly accounted for. In case there is gaseous absorption within the atmosphere, this slightly different angular distribution of the light will lead to a slightly different amount of absorption, and in this case to more absorption and hence deeper absorption lines.

It is important to stress that the decrease of the absolute error in $A_{\mathrm{G}}$ with increasing absorption changes the depth of absorption lines: when polarization is neglected, absorption lines in the numerically simulated $A_{\mathrm{G}}$ are less deep than when polarization is properly included. Using a numerically simulated $A_{\mathrm{G}}$ to derive e.g. mixing ratios of absorbers from an observed $A_{\mathrm{G}}$ will thus lead to mixing ratios that are too high. For example, when one neglects polarization and tries to numerically reproduce the depth of the methane absorption line around $0.73 \mu \mathrm{m}$ (see Fig. 6), thus $A_{\mathrm{G}}$ in the continuum minus $A_{\mathrm{G}}$ in the deepest part of the band, as calculated taking polarization into account, one needs a 1.5 times higher methane mixing ratio (i.e. $0.27 \%$ instead of $0.18 \%$ ). Neglecting polarization when deriving gaseous mixing ratios from planetary observations can thus lead to significant errors.

We also want to recall that the errors shown in Fig. 8 pertain to the geometric albedo of the planet and thus to a total scattering angle $\Theta$ of $180^{\circ}$ (a planetary phase angle $\alpha$ of $0^{\circ}$ ). According to the $\Theta$-dependence of the errors in the planetary phase function in Fig. 3, the effect of neglecting polarization on the depth of absorption lines will generally be different at other values of $\Theta$. In particular, when $\Theta$ is between about $60^{\circ}$ 

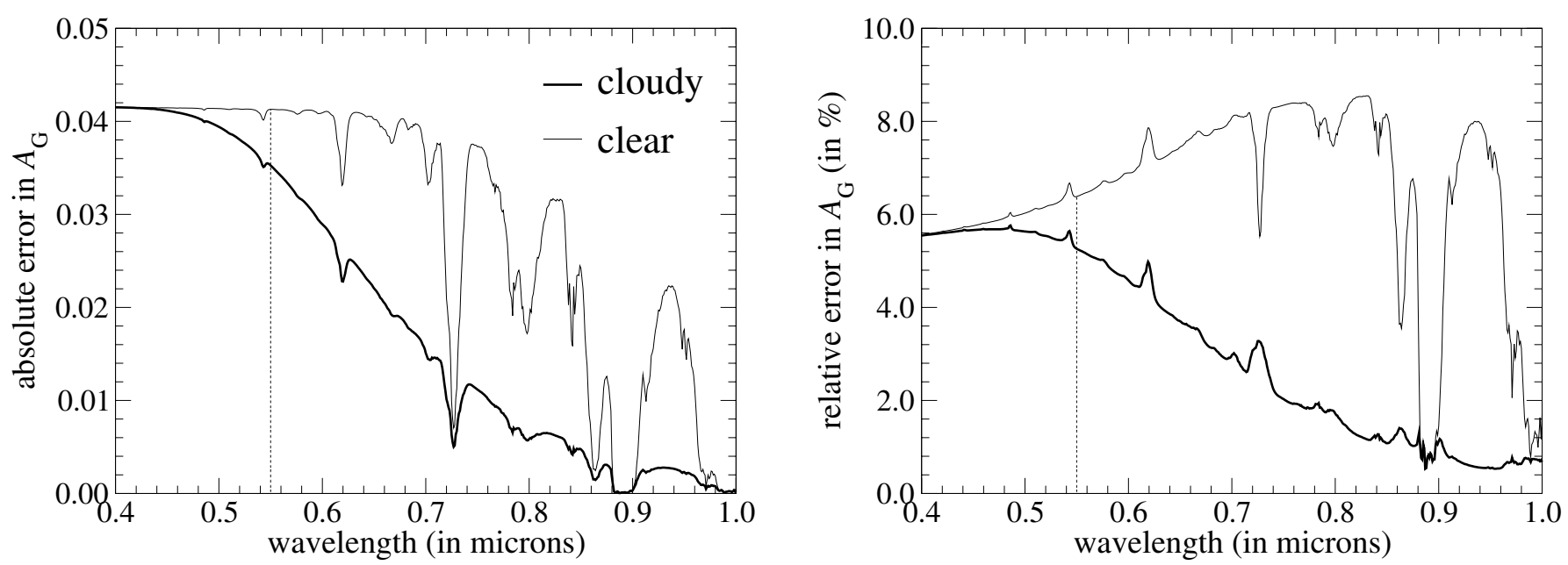

Fig. 8. The absolute a) and relative b) errors in $A_{\mathrm{G}}$ that are due to neglecting polarization. The thin lines pertain to the clear atmosphere (Fig. 6) and the thick lines to the cloudy atmosphere (Fig. 7).
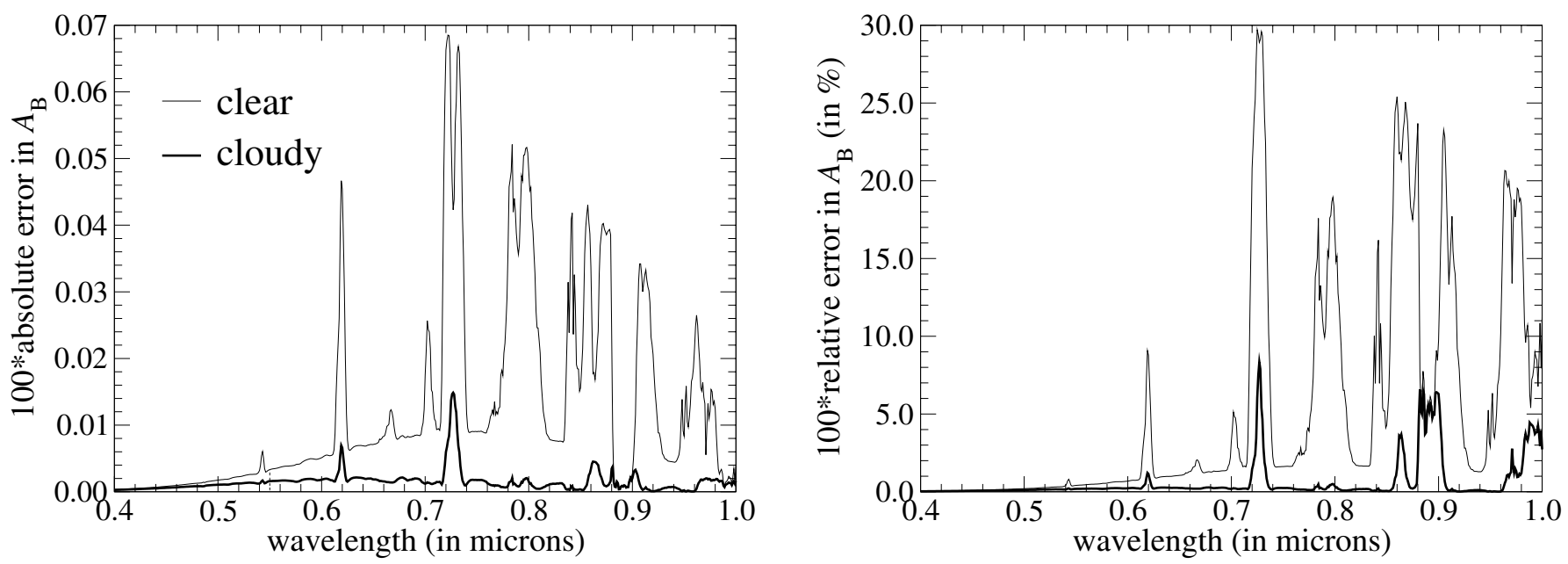

Fig. 9. The absolute a) and relative b) errors in $A_{\mathrm{B}}$ that are due to neglecting polarization. The thin lines pertain to the clear atmosphere (Fig. 6) and the thick lines to the cloudy atmosphere (Fig. 7). Both the absolute and the relative error in the Bond albedo $A_{\mathrm{B}}$ have been multiplied with 100. The maximum absolute error in $A_{\mathrm{B}}$ is thus about 0.0007 for the clear atmosphere, and 0.00015 for the cloudy atmosphere. The maximum relative error is about $0.3 \%$ for the clear atmosphere and about $0.08 \%$ for the cloudy atmosphere.

and $120^{\circ}\left(60^{\circ} \lesssim \alpha \lesssim 120^{\circ}\right)$, which are favourable angles for observations of extrasolar planets, neglecting polarization leads to deeper absorption bands than when polarization is properly accounted for.

Figure 9 shows the wavelength dependent absolute and relative errors in the Bond albedo $A_{\mathrm{B}}$ for the clear model atmosphere and the cloudy model atmosphere (to be discussed in Sect. 3.2.2). Because these errors are very small, they have been multiplied with 100 (just like in Fig. 5) to improve their readability. The wavelength dependence of the continuum in both Figs. 9a and $\mathrm{b}$ is in accordance with what would be expected from Figs. 5a and $\mathrm{b}$, with the maximum errors near $\lambda=0.74 \mu \mathrm{m}$, where the atmospheric molecular scattering optical thickness equals about 1.7.

When comparing the shapes of the absorption bands of the clear model atmosphere in Fig. 9 with those for the clear model atmosphere in Fig. 8, it is apparent that the errors in the deepest absorption bands of $A_{\mathrm{B}}$ are more complex than those in $A_{\mathrm{G}}$. For example, going from the continuum adjacent to the absorption band around $\lambda=0.73 \mu \mathrm{m}$ to the center of the band, the error in $A_{\mathrm{B}}$ first increases, and then decreases again (see Fig. 9a). The decrease of the errors in the deepest parts of absorption bands can be explained by a decrease of multiple scattering. At wavelengths where there is no or little absorption, i.e. the continuum, the shallow absorption bands, and the wings of the deepest absorption bands, the errors are probably predominantly due to the previously discussed slightly different angular distribution of the scattered light within the planetary model atmosphere, and hence to the slightly different amount of absorption when polarization is neglected as compared to when polarization is properly accounted for.

\subsubsection{A cloudy model atmosphere}

Figure 7 is similar to Fig. 6, except that we have added a cloud layer to the planetary model atmosphere. For the calculations 
with polarization, we used $3 \times 3$ matrices, thus including linear polarization but neglecting circular polarization. The cloud layer extends from the atmospheric bottom at an ambient pressure of 5.623 bars to a pressure of 1.0 bars. The cloud particles are described in size by the standard size distribution of Hansen \& Travis (1974), with an effective radius of $1.0 \mu \mathrm{m}$ and an effective variance of 0.1 . The refractive index is 1.42 , and is assumed to be wavelength independent. The cloud layer has a (scattering) optical thickness of 6.0 at $\lambda=0.55 \mu \mathrm{m}$, and its optical properties at other wavelengths are calculated using Mie-theory (as described in Sect. 2.4). For example, the cloud optical thickness increases from 5.8 at $0.4 \mu \mathrm{m}$ to 7.8 at $1.0 \mu \mathrm{m}$.

Comparing Figs. 6 and 7, it appears that at the shortest wavelengths $(\lambda \lesssim 0.45 \mu \mathrm{m})$, adding the cloud layer doesn't increase the planet's geometric and Bond albedos significantly. The reason for this lack of influence is simply that at these wavelengths, the scattering optical thickness of the molecules above the cloud layer is too large for a significant amount of light to first reach the cloud layer and then, after being reflected back towards space, emerge from the atmosphere again. With increasing wavelength, the molecular optical thickness of the atmosphere above the cloud layer decreases, and with that the contribution of light that is reflected by the cloud layer to the total amount of reflected light, and hence to the albedos, increases. Indeed, at the longest wavelengths, the cloudy planet is much brighter than the clear planet (cf. Figs. 6 and 7), at least at continuum wavelengths. For example, at $0.75 \mu \mathrm{m}$, the geometric albedo, $A_{\mathrm{G}}$, of the clear planet is 0.4092 without polarization and 0.4465 with polarization, while that of the cloudy planet is 0.5390 without polarization and 0.5502 with (linear) polarization. In the deepest methane absorption bands, there is little difference between the albedos of the cloudy and the clear planet, because virtually all light is absorbed either before it reaches the cloud layer or after it has been reflected by the cloud layer. In these absorption bands, the cloud layer is thus virtually invisible.

The thick lines in Fig. 8 show the absolute and relative errors in the cloudy planet's geometric albedo $A_{\mathrm{G}}$ when polarization is neglected. Clearly, at the shortest wavelengths, where the contribution of the gaseous atmosphere above the cloud layer dominates the light that is reflected by the planet, the errors due to neglecting polarization for the cloudy model atmosphere are equal to those for the clear model atmosphere. With increasing wavelength, the absolute error in the geometric albedo $A_{\mathrm{G}}$ of the cloudy planet decreases more rapidly than that of the clear model atmosphere, at least at continuum wavelengths (see Fig. 8a). This decrease of the error in $A_{\mathrm{G}}$ is due to a combination of two effects, namely, 1) the contribution of light scattered by cloud particles to the total reflected light increases with wavelength, and 2) the degree of polarization of light that is singly scattered by cloud particles is very low and neglecting polarization thus leads to only small errors. With the decrease of the absolute error in $A_{\mathrm{G}}$ of the cloudy planet at continuum wavelengths (Fig. 8a), the relative error in $A_{\mathrm{G}}$ decreases, too (Fig. 8b).

In the methane absorption bands, the absolute errors in $A_{\mathrm{G}}$ of the cloudy atmosphere are smaller than in the adjacent continuum (Fig. 8a). Like for the clear model atmosphere, neglecting polarization thus leads to absorption bands that are less deep than when polarization is properly accounted for. For the cloudy atmosphere, the relative errors in all but the deepest of the methane absorption bands (i.e. that around $0.89 \mu \mathrm{m}$ ) are larger than in the adjacent continuum (Fig. 8b). This implies that the slightly different angular distribution of the scattered light in the atmosphere that is due to neglecting polarization results in slightly more absorption and thus deeper absorption lines than when polarization is taken into account. This effect was also seen for the clear atmosphere (cf. the thin line in Fig. 8b), except only for the absorption bands at wavelengths shorter than about $0.7 \mu \mathrm{m}$. As explained in Sect. 3.2.1, in absorption bands at longer wavelengths, the errors for the clear atmosphere should mostly be due to a decrease of multiple scattering. For the cloudy atmosphere, the decrease of multiple scattering would only be a source of errors for the deepest absorption band, because the scattering optical thickness of the cloudy atmosphere is much larger than that of the clear atmosphere, in particular at the longer wavelengths.

The geometric albedo $A_{\mathrm{G}}$, discussed above, pertains to a total scattering angle $\Theta$ of $180^{\circ}$ (or a planetary phase angle $\alpha$ of $0^{\circ}$ ). Like with a clear planetary atmosphere, the errors in the flux that is reflected by the cloudy planet that are due to neglecting polarization will generally depend on $\Theta$. This is illustrated in Fig. 10 for three different wavelengths, namely $0.55 \mu \mathrm{m}, 0.75 \mu \mathrm{m}$, and $0.95 \mu \mathrm{m}$ (all continuum wavelengths). Note that this figure shows the errors in the phase function $a_{1}$ (which equals $4 A_{\mathrm{G}}$ at $\Theta=180^{\circ}$ ). For the cloudy planet, the $\Theta$-dependence of the errors has a similar shape as that for the clear planet (cf. Fig. 3), with the largest (relative) errors occuring around $\Theta=20^{\circ}, 90^{\circ}$, and $180^{\circ}$.

The thick lines in Fig. 9 show the absolute and relative errors in the cloudy planet's Bond albedo $A_{\mathrm{B}}$ when polarization is neglected. Like for the clear atmosphere, these errors are very small and therefore we have multiplied them by 100 in the figure. Although difficult to see in Fig. 9, at the shortest wavelengths the errors for the cloudy model atmosphere are equal to those for the clear model atmosphere, because the light that is reflected by the planet is dominated by the contribution of the gaseous atmosphere above the cloud layer (cf. Fig. 8). The continuum errors in $A_{\mathrm{B}}$ of the cloudy planet are much smaller than those of the clear planet (both the absolute and the relative errors). This is fully expected, because the thick cloud layer prevents virtually all light to reach the black surface below the atmosphere where it would be absorbed. Thus, in the absence of absorption in the atmosphere, almost all light that is incident on the cloudy planet is reflected back to space, whether polarization is taken into account in the radiative transfer calculations or not.

In the methane absorption bands, the errors in $A_{\mathrm{B}}$ for the cloudy planet are generally smaller than those for the clear planet. A close look at the precise structure of individual bands reveals a structure at least as complicated as that for the clear atmosphere, revealing a delicate wavelength dependent balance between gaseous absorption, scattering by molecules and scattering by cloud particles. We refrain from attempting to unravel this balance because the errors are extremely small; 

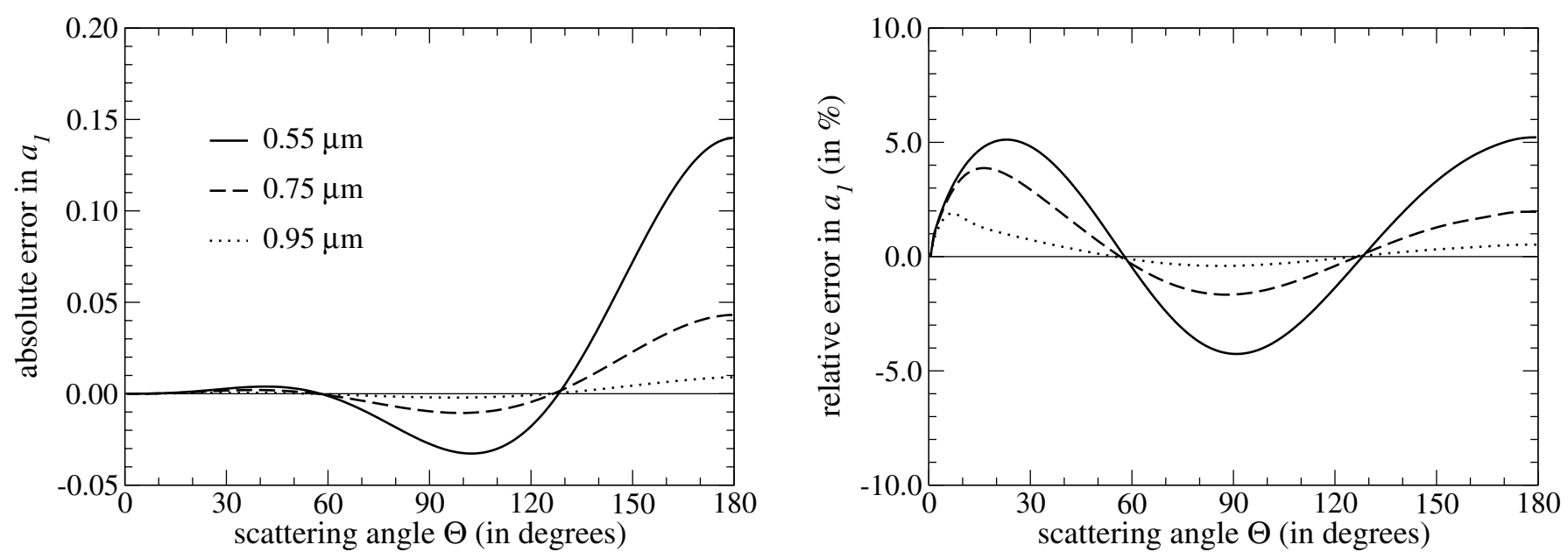

Fig. 10. The errors in the phase functions of the cloudy model planet when polarization is neglected: a) the absolute errors, and b) the relative errors. The errors are shown at the following wavelengths: $0.55 \mu \mathrm{m}$ (solid line), $0.75 \mu \mathrm{m}$ (dashed line), and $0.95 \mu \mathrm{m}$ (dotted line).

the maximum absolute error is namely 0.00015 and the maximum relative error $0.08 \%$ (both at $\lambda=0.73 \mu \mathrm{m}$ ).

For an atmosphere containing aerosol and/or cloud particles, it will in principle matter whether all polarization, thus linear and circular $(1 \times 1$ versus $4 \times 4$ matrices $)$, is neglected, or only the circular polarization $(1 \times 1$ versus $3 \times 3$ matrices $)$. To investigate the errors resulting from neglecting only circular polarization, thus using $3 \times 3$ matrices instead of $4 \times 4$, we have performed numerical simulations for the cloudy, Jupiterlike model atmosphere using $4 \times 4$ matrices. These simulations show that planetary phase functions and albedos calculated including only the linear polarization differ by less than $0.0005 \%$ from those calculated including both the linear and the circular polarization.

\section{Summary and discussion}

Our numerical simulations for clear and cloudy Jupiter-like model planets show that when light is described by a scalar instead of by a vector, the error in the calculated phase function of a planet varies strongly with the total scattering angle $\Theta$ (and thus with the planetary phase angle $\alpha$ ) and can be on the order of several percent. The largest absolute errors in the phase function are generally found at $\Theta=180^{\circ}\left(\alpha=0^{\circ}\right)$, and thus in the geometric albedo, $A_{\mathrm{G}}$, of the planet. In particular for clear model atmospheres, the error in the geometric albedo can reach almost $9 \%$ (with the planet being darker when polarization is neglected). Note that although neglecting polarization leads to the largest absolute errors for $\Theta=180^{\circ}$, the degree of polarization of light reflected by a planet itself is zero for $\Theta=180^{\circ}$ (see e.g. Stam et al. 2004).

The error in the planetary Bond albedo is generally much smaller than that in the geometric albedo, namely, it is typically smaller than $0.5 \%$. The error in the calculated Bond albedo due to neglecting polarization is much smaller than the errors that can reasonably be expected for Bond albedo measurements. Consequently, in case one is only interested in planetary Bond albedos, polarization can safely be neglected.
When describing molecular scattering in our simulations, we used a depolarization factor representative for $\mathrm{H}_{2}$, the main component of Jupiter-like atmospheres, namely 0.02 . Mishchenko et al. (1994) found that for plane-parallel model atmospheres, thus not integrated over the planetary disk, errors in the planetary phase function due to neglecting polarization generally decrease with increasing depolarization factor. For comparison, the maximum error (considering all scattering geometries and atmospheric scattering optical thicknesses) reported by Mishchenko et al. (1994) for a clear, non-absorbing plane-parallel model atmosphere with a black surface below is $12 \%$ when the depolarization factor is 0.0 , and about $8 \%$ when the depolarization factor is 0.086 . According to Sromovsky (2005b), the absolute error in the geometric albedo of a planet with a clear, semi-infinite atmosphere due to neglecting polarization is larger by a value of 0.005 when the depolarization factor is 0.0 instead of 0.022 . Although the value of the depolarization factor is thus important for the error in the reflected flux due to neglecting polarization, it does not change it drastically.

We find that more than on the depolarization value, do the errors due to neglecting polarization depend on the atmospheric absorption optical thickness. Neglecting polarization thus clearly changes both the shape and, more importantly, the depth of gaseous absorption bands. As a result, if one neglects polarization in radiative transfer calculations when deriving e.g. gaseous mixing ratios from the observed depths of gaseous absorption bands, errors of several tens of percent can result.

It is important to note here that the methane absorption cross-sections that were used in this paper, have been derived from the depths of methane absorption bands in observed geometric albedo spectra of the Jovian planets (see Karkoschka 1994). As far as we know, this derivation did not include polarization. The absorption cross-sections will thus differ from the real ones. For our purposes, i.e. showing that neglecting polarization leads to errors in calculated fluxes, we don't need accurate values of absorption cross-sections. However, using hence derived cross-sections in the interpretation of observations of an arbitrary planet could give rise to unexpected errors (except 
of course when this planet is observed at the same phase angle and when it has an atmosphere similar to the one the absorption cross-sections were derived for).

The results presented in this paper suggest that at least for planetary model atmospheres containing spherical particles and/or irregularly shaped particles in random orientation, describing light by its intensity or flux and state of linear polarization should suffice for most applications, and the circular polarization can be neglected. Namely, the errors due to neglecting only the circular polarization (thus using $3 \times 3$ matrices instead of $4 \times 4$ matrices) are generally smaller than $0.0005 \%$ (recall that for atmospheres containing only gaseous molecules, the results for $3 \times 3$ matrices are identical to those for $4 \times 4$ matrices). This is very fortunate because using $3 \times 3$ instead of $4 \times 4$ matrices saves significant amounts of computing time. When wavelength dependent numerical simulations are required, two calculations, one using $3 \times 3$ matrices and the other using $4 \times 4$ matrices at a wavelength where the scattering optical thickness of gaseous molecules is small compared to that of the aerosol and/or cloud particles could help to establish whether the error due to neglecting circular polarization is small enough for the purpose.

\section{References}

Chandrasekhar, S. 1950, Radiative transfer (Oxford: Clarendon Press) Charbonneau, D., Allen, L. E., Megeath, S. T., et al. 2005, ApJ, 626, 523

Chauvin, G., Lagrange, A.-M., Dumas, C., et al. 2004, A\&A, 425, L29
Chauvin, G., Lagrange, A.-M., Zuckerman, B., et al. 2005, A\&A, 438, L29

de Haan, J. F., Bosma, P. B., \& Hovenier, J. W. 1987, A\&A, 183, 371

de Rooij, W. A., \& van der Stap, C. C. A. H. 1984, A\&A, 131, 237

Deming, D., Seager, S., Richardson, L. J., \& Harrington, J. 2005, Nature, 434, 740

Hansen, J. E. 1971, J. Atmospheric Sciences, 28, 1400

Hansen, J. E., \& Travis, L. D. 1974, Space Sci. Rev., 16, 527

Hovenier, J. W., \& Stam, D. M. 2005, J. Quantitative Spectroscopy and Radiative Transfer (in press)

Hovenier, J. W., van der Mee, C., \& Domke, H. 2004, Transfer of Polarized Light in Planetary Atmospheres; Basic Concepts and Practical Methods (Dordrecht: Kluwer; Berlin: Springer)

Karkoschka, E. 1994, Icarus, 111, 174

Kemp, J. C., Henson, G. D., Steiner, C. T., \& Powell, E. R. 1987, Nature, 326, 270

Lacis, A. A., Chowdhary, J., Mishchenko, M. I., \& Cairns, B. 1998, Geophys. Res. Lett., 25, 135

Lindal, G. F. 1992, AJ, 103, 967

Mishchenko, M. I., Lacis, A. A., \& Travis, L. D. 1994, J. Quantitative Spectroscopy and Radiative Transfer, 51, 491

Sromovsky, L. A. 2005a, Icarus, 173, 254

Sromovsky, L. A. 2005b, Icarus, 173, 284

Stam, D. M., Aben, I., \& Helderman, F. 2002, J. Geophys. Res. D (Atmospheres), AAC 1

Stam, D. M., Hovenier, J. W., \& Waters, L. B. F. M. 2004, A\&A, 428, 663

van de Hulst, H. C. 1957, Light Scattering by Small Particles (New York: J. Wiley and Sons)

van de Hulst, H. C. 1980, Multiple Light Scattering, Tables, Formulas, and Applications, Vols. 1 and 2 (New York: Academic Press)

West, R. A., Strobel, D. F., \& Tomasko, M. G. 1986, Icarus, 65, 161 